
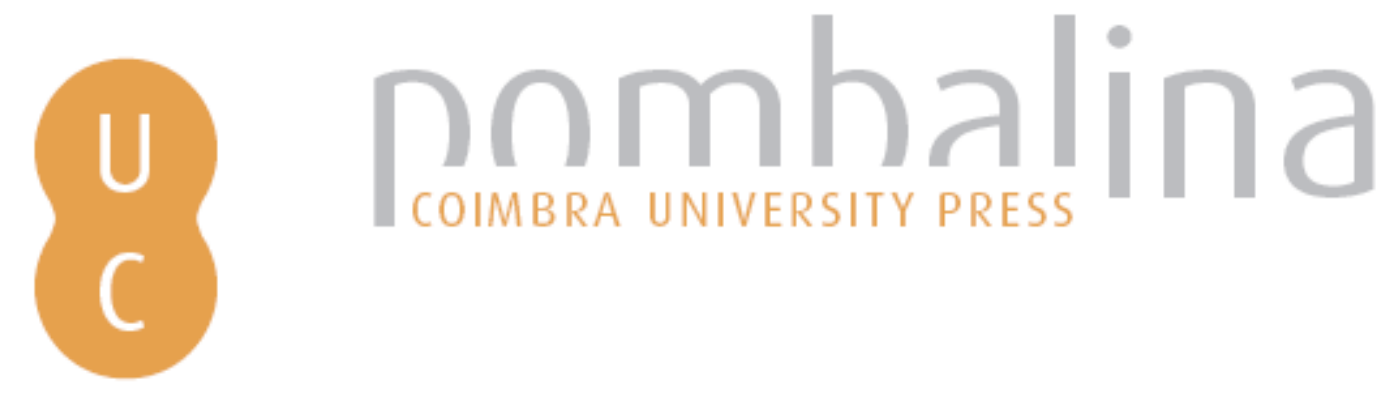

\title{
Integração dos sistemas de registro e cadastro através de um sistema de informações geográficas
}

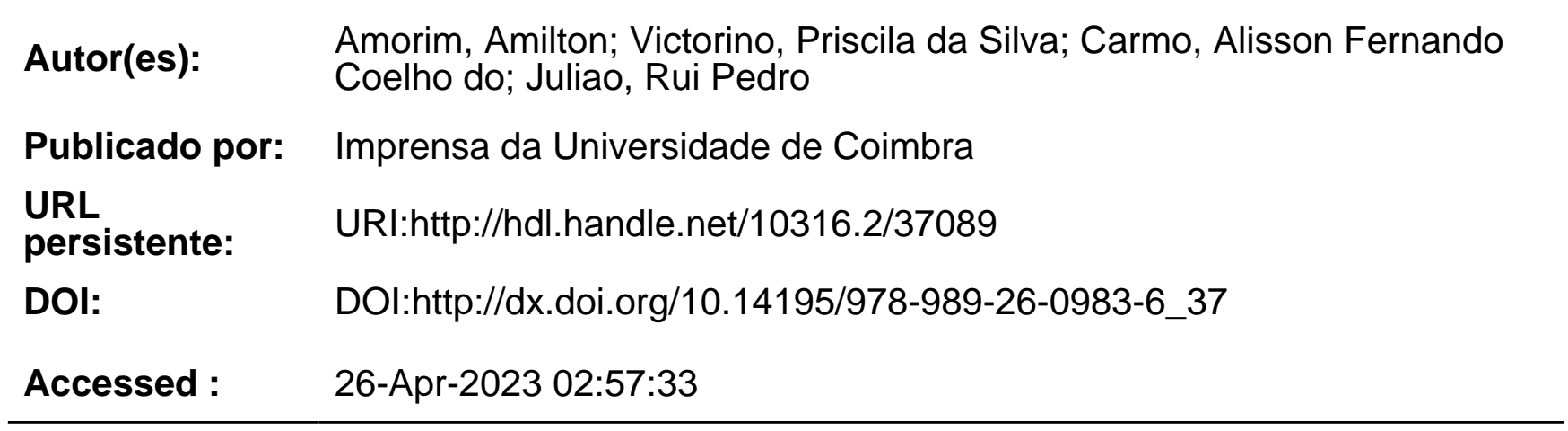

A navegação consulta e descarregamento dos títulos inseridos nas Bibliotecas Digitais UC Digitalis, UC Pombalina e UC Impactum, pressupõem a aceitação plena e sem reservas dos Termos e Condições de Uso destas Bibliotecas Digitais, disponíveis em https://digitalis.uc.pt/pt-pt/termos.

Conforme exposto nos referidos Termos e Condições de Uso, o descarregamento de títulos de acesso restrito requer uma licença válida de autorização devendo o utilizador aceder ao(s) documento(s) a partir de um endereço de IP da instituição detentora da supramencionada licença.

Ao utilizador é apenas permitido o descarregamento para uso pessoal, pelo que o emprego do(s) título(s) descarregado(s) para outro fim, designadamente comercial, carece de autorização do respetivo autor ou editor da obra.

Na medida em que todas as obras da UC Digitalis se encontram protegidas pelo Código do Direito de Autor e Direitos Conexos e demais legislação aplicável, toda a cópia, parcial ou total, deste documento, nos casos em que é legalmente admitida, deverá conter ou fazer-se acompanhar por este aviso.

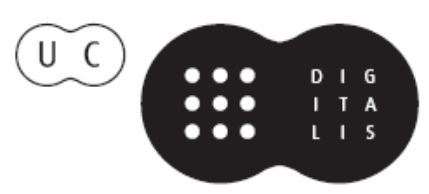




\section{$\forall$ \\ TAS DAS I JORNADAS LUSÓFONAS DE CIÊNCIAS E TECNOLOGIAS DE INFORMAÇÃO GEOGRÁFICA}

Editores

José Gomes dos Santos

Cidália Fonte

Rui Ferreira de Figueiredo

Alberto Cardoso

Gil Gonçalves

José Paulo Almeida

Sara Baptista 


\title{
A RTIGO 37 \\ INTEGRAÇÃO DOS SISTEMAS DE REGISTRO E CADASTRO ATRAVÉS DE UM SISTEMA DE INFORMAÇÕ ES GEOGRÁFICAS
}

\author{
AMORIM, Amilton ${ }^{1}$; VICTORINO, Priscila da Silva \\ CARMO, Alisson Fernando Coelho do 3 E JULIÃO, Rui Pedro ${ }^{4}$
}

\begin{abstract}
1,2,3 Universidade Estadual Paulista - UNESP, Faculdade de Ciências e Tecnologia; Programa de Pós-Graduação em Ciências Cartográficas; Departamento de Cartografia - Tel.: +551832295500; Rua Roberto Simonsen, 305. CEP- 19060-900 - Presidente Prudente - SP; amorim@fct.unesp.br ; priscila.svictorino@ hotmail.com; alisondocarmo@gmail.com

${ }^{4}$ e-GEO-FCSH/UNL; Avenida de Berna, 26-C 1069-061 Lisboa; Tel: +351 2179083 00; email: rpj@fcsh.unl.pt
\end{abstract}

\section{RESUMO}

O avanço da informática, o desenvolvimento dos Sistemas de Informações Geográficas e o surgimento de profissionais capacitados para atuar na área, revelam às prefeituras e aos serviços registrais novas possibilidades de intercâmbio de informações entre estas duas instituições. Tal fato permite uma melhor descrição da realidade legal e geométrica dos imóveis, além de possibilitar que esses dados sejam compartilhados entre outros sistemas computacionais e disponibilizados aos usuários em formato digital. Dessa forma, este trabalho consiste em investigar estratégias para a integração entre os sistemas de Cadastro e Registro de Imóveis, utilizando um Sistema de Informações Geográficas desenvolvido para este propósito, por meio de tecnologias para disponibilização de mapas interativos na Internet. Este método, ainda em desenvolvimento neste protótipo, considera a necessidade dessas áreas em adquirir informações contidas em ambos os sistemas, bem como o aperfeiçoamento da gestão administrativa na tomada de decisões. Os primeiros resultados 
apontam para uma melhoria do atendimento ao usuário, agilizando o processo na solicitação de informações referentes aos imóveis.

\title{
PALAVRAS-CHAVE
}

SIG Cadastral, Cadastro técnico multifinaliltário, Integração entre os sistemas de cadastro e registro.

\section{INTEGRATION BETWEEN SYSTEMS CADASTRE AND REGISTRY}

THROUGH A GEOGRAPHIC INFORMATION SYSTEM

\begin{abstract}
The advancement of computer technology, the development of Geographic Information Systems and the emergence of professionals qualified to work in this area, have shown to county and registry services new possibilities for information exchange between these two institutions. This fact allows a better description of the legal and geometric reality of the properties, and it also enables these data to be shared between other computing systems and made available to users in digital format. So, this work consist in investigate methods of integration between the Cadastre and Registration systems using Geographic Information Systems (GIS) developed for this purpose, through the technologies for interactive maps on the Internet. This method, still under development in this prototype, considers the need for these areas to acquire information contained in both systems, the improvement of administrative management in decision making. The first results indicate an improvement of customer service, streamlining the process in the solicitation of information related to real estate.
\end{abstract}

\section{KEYWORDS}

Cadastral GIS, Multipurpose technical cadastre, Integration between systems cadastre and registry.

\section{INTRODUÇÃO}

Apesar de ser uma atividade muito antiga, a Cartografia, que serviu como importante instrumento de navegação e registro de terras ao Homem por muitos anos, chegou a um ponto que não bastava mais ter feições do espaço objeto representadas analogicamente, mesmo tendo evoluído da rocha ao papel. As exigências começaram a ser cada vez maiores, de acordo com a apresentação de novas tecnologias que foram desenvolvidas 
a partir do avanço tecnológico, principalmente, nas áreas de Eletrônica e Informática.

Com o avanço tecnológico nessas áreas, surgiu a possibilidade de se informatizar os mapeamentos (digital) e ainda acoplar dados aos mesmos, por meio de Banco de Dados (atributos), juntamente, novas demandas e anseios surgiram.

O avanço tecnológico em Cartografia, nas duas últimas décadas, foi muito forte quando comparado com o avanço no último século. Logo após o surgimento dos sistemas computacionais de desenho, os chamados CAD (Computer Aided Design), surgiram os Bancos de Dados Geográficos (BDG) com aplicações, até então, apenas sonhadas por pesquisadores.

Quando se fala em Cadastro Territorial Multifinalitário (CTM), não se pode deixar de falar em Cartografia, que é base de sua construção e modelagem, e muito menos na inovação tecnológica quem vem viabilizando a execução de trabalhos inovadores nesta área.

Por outro lado, o mesmo avanço tecnológico que tem viabilizado projetos nesta área, principalmente pelo desenvolvimento de instrumentos inovadores e redução de custos, também tem provocado uma grande preocupação com o uso indiscriminado de instrumentos inadequados para a implantação de sistemas cadastrais, em razão da facilidade de acesso que se tem a produtos cartográficos de baixo custo ou até gratuitos.

O Sistema Registral da Propriedade visa dar publicidade, autenticidade e segurança aos atos jurídicos, portanto pode-se dizer que o Registro de Imóveis não registra o imóvel propriamente dito, mas sim os direitos relativos a eles. O direito de propriedade é abstrato, não sendo palpável, ou seja, não é o imóvel, e sim o "direito" que uma ou mais pessoas detém sobre o bem cadastrado e adequadamente descrito (RAMBO, 2011).

Uma das principais tendências mundiais que vem sendo observada, além da utilização de informações cadastrais para múltiplas finalidades, é a utilização dessas para resolver problemas de descrição dos imóveis, fortalecendo um dos princípios registrais (especialidade), que tem por objetivo a descrição inequívoca dos imóveis para individualizar os registros. No entanto, sabe-se que essa descrição, que sempre ficou a cargo 
do próprio sistema de registro de imóveis, nem sempre consegue se apresentar como "inequívoca".

Acredita-se que se possa contribuir significativamente para a minimização desses problemas, utilizando o Cadastro Territorial Multifinalitário como um sistema de disponibilização de informações que podem ser utilizadas diretamente pelo sistema de registro de imóveis.

Pretende-se assim mostrar alguns resultados, obtidos até o momento, no sentido de se propor um método para a interconexão do sistema cadastral de uma cidade de pequeno porte com o sistema de registro de imóveis, utilizando ferramentas de Sistema de Informações Geográficas (SIG), implementado a partir de dados cadastrais dos imóveis que, até então, são utilizados apenas para fins tributários.

Para que seja viável a interconexão do Cadastro com o Registro de Imóveis serão necessárias algumas adaptações no atual sistema cadastral, principalmente no que diz respeito ao rol de informações disponíveis e sua compatibilização com as necessidades do sistema de registro de imóveis.

Acredita-se que, com a utilização do Sistema de Informações Geográficas desenvolvido a partir de dados cadastrais, os procedimentos de registro serão melhorados, principalmente pelo fato do atendimento ao princípio registral da especialidade ser simplificado.

Neste contexto, este trabalho visa investigar os aspectos envolvidos na modelagem e desenvolvimento de um Sistema de Informações Geográficas que auxilie na integração entre as informações dos sistemas de Cadastro Territorial Multifinalitário e Registro de imóveis de um município. Dessa forma, objetiva-se que a implementação dos mapas interativos e dinâmicos, disponibilizados na Internet possa auxiliar o acesso dos usuários a estas informações de forma simples e intuitiva.

\section{FUNDAMENTAÇÃo TEÓRICO-METODOLÓGICA}

\subsection{Interconexão entre o Cadastro Territorial Multifinalitário e Registro} de Imóveis 
O Cadastro Territorial Multifinalitário e o Registro de Imóveis são instituições diferentes com objetivos distintos, porém ambos possuem dados e informações referentes ao mesmo objeto, que é o imóvel. O registro de imóveis exerce o controle sobre informações a respeito do proprietário do imóvel e a forma de aquisição do mesmo, enquanto o Cadastro realiza, entre outras funções, a atividade de definir a localização geográfica da parcela territorial e seu respectivo valor venal.

Segundo Jacomino (2000 apud CARNEIRO, 2003) "a Lei de Registros Públicos estabelece que os imóveis matriculados devem estar especializados, ou seja, perfeitamente descritos e caracterizados, com todas as minudências que permitam individuá-los e estremá-los de quaisquer outros”.

Assim, para garantir o princípio registral da especialidade é necessário, de acordo com Carneiro (2003), que "o Cadastro forneça ao Registro de imóveis os dados descritivos e gráficos dos imóveis, especialmente medidas lineares, superficiais e limites, como também os dados que os individualizem dentro dos mapas oficiais. Porém, é preciso que o Registro Imobiliário, por sua vez, forneça ao Cadastro as modificações das titularidades de domínio dos imóveis, derivadas de atos de disposição por vontade do titular".

O processo de intercâmbio de informações, entre essas duas entidades, deve ser cuidadosamente elaborado para que seja assegurada a unicidade de cada propriedade e evite a superposição de áreas. A tarefa da determinação da localização geográfica da parcela territorial contribui para que esta seja descrita com todos os seus limites e confrontações, considerando o princípio da especialidade. Para tanto, é importante que as bases e dados cartográficos estejam georreferenciados, ou seja, referenciados ao Sistema Geodésico Brasileiro.

Atualmente, existem softwares que viabilizam o desenvolvimento de Sistemas de Informações Geográficas voltados à Web, muitos gratuitos e de código aberto (Software Livre). Tais tecnologias podem ser utilizadas na criação de mapas dinâmicos, por meio de consultas espaciais baseadas nas necessidades do usuário. Esta forma avançada de difusão e interligação de dados é um importante recurso que pode facilitar o acesso às informações relacionadas ao cadastro imobiliário urbano municipal e 
contribuir com o processo de modernização da gestão pública.

Mesmo com o frequente desenvolvimento e avanço dos recursos tecnológicos, muitos órgãos públicos tem dificuldades para promover a integração das informações que pertencem a um mesmo contexto, mas com origens e finalidades diferentes, como é o caso das informações provenientes do Cadastro Territorial Multifinalitário e o Registro de Imóveis. É comum encontrar duplicidades e ambiguidade das informações em razão da individualização dos métodos de manipulação, muitas vezes manuais, ou seja, não informatizados.

\subsection{Sistema de Informação Territorial - SIT}

Comumente confundido com um simples Sistema de Informações Geográficas, o SIT possui características que o diferenciam de um SIG, principalmente pelo fato de ser um sistema baseado em parcelas, que registra direitos, restrições e responsabilidades, podendo ser desenvolvido para múltiplas finalidades, utilizando dados do Cadastro Territorial Multifinalitário, tornando-se um importante instrumento de apoio ao planejamento (AMORIM \& YAMASHITA, 2008; FIG, 2010).

Um SIG pode ser implementado como um sistema para captura, armazenamento, verificação, integração, análise e apresentação de dados sobre a Terra, espacialmente referenciados. Obrigatoriamente, inclui um banco de dados georreferenciados e outros softwares apropriados. Um SIT é um sistema de aquisição, processamento, armazenamento e distribuição de informações sobre a terra. Assim pode-se entender que um SIG pode ser utilizado como ferramenta para implementação de um SIT (SASS \& AMORIM, 2013; WILLIAMSON et al. ,2010).

De acordo com os Artigos $4^{\circ}$ e $5^{\circ}$, da Portaria MC - 511/2009, o SIT é formado pelos dados do CTM relacionados ao Registro de Imóveis e acrescentados os dados dos cadastros temáticos (BRASIL, 2009).

De acordo com SASS \& AMORIM (2013), essas definições nos levam a entender que um Cadastro Territorial Multifinalitário informatizado dá origem ao SIT, composto de hardware, software, pessoas, dados e infraestrutura de rede, com o objetivo de gerenciar o CTM, e possivelmente o 
Registro de Imóveis, além de disponibilizar dados alfanuméricos e gráficos para apoiar a gestão territorial.

Tão importante quanto a implementação deve-se considerar os processos de atualização, processamento e distribuição dos dados espaciais para o apoio à tomada de decisão (LARSSON, 1996; SASS \& AMORIM, 2013).

Considerando a disponibilização de dados e informações, um SIT deve ser projetado para prever formas de fornecimento de dados como produtos que, inclusive possam ser comercializados (como mapas e documentos descritivos), ou em forma de serviços especificamente solicitados. Sendo assim, espera-se que um SIT possa fornecer atributos, que podem ser apresentados de forma textual ou numérica e dados espaciais, que podem ser apresentados em mapas (DALE \& MCLAUGHLIN, 1990).

\subsection{Sistema Gerenciador de Banco de Dados - SGBD}

Um banco de dados consiste em um conjunto de dados inter-relacionados e organizados de forma que seus dados possam ser facilmente manipulados. Esta é a maneira mais viável de armazenar uma grande quantidade de dados, como por exemplo, os dados cadastrais de um município (DALAQUA et al., 2004).

Segundo Date (2000), o SGBD é a ferramenta que conecta o usuário ao banco de dados, ou seja, todas as intervenções feitas pelo usuário ao banco de dados são feitas por meio do SGBD. Tais intervenções podem ser descritas como, acrescentar e remover arquivos ou tabelas, e também buscar e atualizar dados. Dentre os SGBDs atuais mais popularmente utilizados, temos o PostgreSQL.

O PostgreSQL, utilizado neste trabalho, é um SGBD objeto-relacional de código aberto, robusto, confiável e flexível. É considerado objeto-relacional por implementar, além das características de um SGBD relacional, algumas características de orientação a objetos, como herança e tipos personalizados. O PostgreSQL possui recursos como: consultas complexas, chaves estrangeiras, integridade transacional, controle de concorrência multi-versão, suporte ao modelo híbrido objeto-relacional, entre outros (POSTGRESQLBR, 2014). 
A linguagem SQL é uma linguagem de definição e manipulação de dados, isto é, com a referida linguagem podemos definir e construir tabelas/entidades, e também manipular diversas relações para a obtenção dos resultados desejados (MALAMAN \& AMORIM, 2010).

\subsection{Servidor de mapas Web Services}

Os servidores de mapas permitem aos usuários a interação com a informação espacial, possibilitando o acesso às informações no formato original, e consequentemente, consultas em diferentes níveis de complexidades (PARMA, 2006).

Segundo Miranda (2003) para possibilitar a publicação de mapas pela Web, alguns aspectos devem ser analisados, pois algumas funções básicas de SIG, como mudanças de escala, navegação, consulta ao banco de dados, interação com o mapa, entre outras, precisam ser implementadas.

O MapServer, utilizado neste trabalho, pode ser descrito como um conjunto de recursos e ferramentas de código aberto (Open Source) que permite o desenvolvimento de aplicações geográficas em ambientes de Internet e Intranet. Ele foi desenvolvido com a utilização de outros projetos de software livre sob patrocínio da $N A S A$ (EUA) a partir de 1996. Sua plataforma de operação compreende aos sistemas Unix-like e Windows. Este sistema é o servidor de mapas de código aberto mais popular na área das geotecnologias e tem seu uso bastante difundido ao redor do mundo (MIRANDA, 2002).

MapServer é um Web service que pode ser implementado como um sistema individual CGI (Common Gateway Interface) ou como módulo interno de linguagens de programação, como PHP, a partir da utilização da biblioteca MapScript, sendo possível assim, utilizar seus recursos dentro do contexto de execução do ambiente de programação.

Este sistema, oferece recursos para a construção e disponibilização de mapas a partir de consultas em diferentes tipos de bases de dados, como um SGBD - dados armazenados em um banco de dados controlados por SGBD - ou Shapefile - dados armazenados em um arquivo com definições geoespaciais. 
As consultas e definição dos mapas são direcionadas a partir de um arquivo declarativo nomeado de mapfile. O mapfile armazena informações para a construção das camadas de representação do mapa e pode ser construído dinamicamente, a partir de uma linguagem de programação como o PHP.

\subsection{XML (eXtensible Markup Language)}

A crescente utilização de Web services, e consequente integração de diferentes tecnologias, exige a definição de um padrão de comunicação a ser respeitado, independente da tecnologia utilizada. Este é o propósito do XML: oferecer uma estrutura padronizada para a representação de qualquer conteúdo, para favorecer a integração de sistemas.

O XML é uma linguagem de marcação de texto que pode ser utilizada para descrever diversos tipos de dados, de forma hierárquica, com o objetivo de facilitar a distribuição de dados, principalmente pela Internet. XML é independente de plataforma, tanto de hardware como de software, permitindo que aplicações distintas sejam capazes de manipular o mesmo conjunto de dados definidos em um arquivo XML por meio de operações de leitura e escrita do arquivo.

Esta também é uma linguagem utilizada como base para outras linguagens, ressaltando sua flexibilidade e capacidade de extensão, como por exemplo, o XHTML (formato para páginas Web), o MathML (formato para expressões matemáticas), SVG (formato para gráficos vetoriais), GML (formato para dados geográficos), entre outros (OGC, 2011).

\subsection{Integração de Tecnologias de Desenvolvimento WEB}

A utilização de serviços e ferramentas voltadas para o desenvolvimento de mapas digitais na Web tem crescido ao longo dos tempos. Existem diversas ferramentas disponíveis para a construção e disponibilização de mapas no ambiente Web, como aquelas abordadas por Schmidt e Weiser (2012), os quais apresentam uma evolução histórica dos serviços de Web Mapping (disponibilização e acesso de mapas no ambiente Web) e concluem afirmando sobre a forte tendência de transformação dos mapas, 
a priori construídos de forma estática, para sua concepção dinâmica, referente tanto à elaboração colaborativa dos mapas quanto ao consumo do conteúdo inseridos no mapa.

A quantidade crescente de serviços de Web Mapping disponíveis pode ser conferida à constante evolução e disponibilização de ferramentas que permitem a manipulação destes objetos, como bancos de dados espaciais, servidores e gerados de mapas, visualizadores, entre outros. No entanto, para permitir a integração entre os diferentes componentes que viabilizam a construção do contexto de um SIG ou SIT, é necessária a utilização de mecanismos capazes de estabelecer a comunicação entre os mesmos, papel este, desempenhado por linguagens de programação que efetuam a ligação entre os componentes.

Basicamente, assim como as camadas de apresentação, aplicação e de dados que definem a arquitetura genérica proposta por Luaces et al. (2004) para a construção de um SIG, o fluxo de execução de sistemas destas categorias é geralmente baseado na utilização de um visualizador que exibe um mapa construído dinamicamente por meio de processamento e consulta à uma base de dados espacial. Como o foco de um SIG ou SIT é o contexto Web, a construção da interface de visualização deve ser baseada em tecnologias que possam favorecer sua disponibilização e execução em um navegador de Internet.

Uma das abordagens, e a que foi utilizada neste projeto, consiste na construção da interface de visualização utilizando a linguagem de marcação HTML (linguagem para construção de páginas Web) integrada com a capacidade de interação oferecida pela linguagem de scripts Javascript que pode realizar requisições assíncronas de dados, por meio de AJAX, para a camada de processamento desenvolvida em PHP. PHP é uma linguagem de programação robusta voltada, principalmente, para o desenvolvimento de sistemas e aplicativos Web. Sua principal característica reside no fato de ser executada no ambiente do servidor Web (Server-side), oferecendo o cenário ideal para a construção de Web services, ou seja, sistemas que oferecem serviços de processamento, disponíveis a outras aplicações através da Web. 
Uma vez que a camada de processamento recebe uma requisição, esta efetua o tratamento dos dados e realiza a consulta a um banco de dados espacial, como o PostgreSQL com a extensão PostGIS, para que no final do ciclo, as informações retornadas do banco de dados possam ser representadas em um mapa construído dinamicamente por um servidor ou gerador de mapas, como o MapServer.

\section{PROCEDimentos metodológicos}

Para o desenvolvimento deste trabalho foi utilizado o levantamento cadastral de 2010, executado por Malaman e Amorim (2010) no município de Ribeirão dos índios - SP.

Os dados cadastrais se referem às características físicas e geométricas dos imóveis, relacionadas com os atributos dos respectivos proprietários. Dessa forma, pôde-se desenvolver os aplicativos a partir dos dados reais, que podem ser utilizados para as duas finalidades de interesse deste trabalho (Cadastro e Registro), uma vez que o Banco de Dados Cadastrais contempla a maioria dos dados necessários para o referido desenvolvimento.

\subsection{Desenvolvimento}

\subsubsection{Modelagem do banco de dados}

A primeira etapa para a concepção de um sistema é o planejamento da modelagem dos dados de entrada. Especificamente neste trabalho, existem dois principais aspectos envolvidos relacionados ao armazenamento dos dados: modelagem das informações relacionadas ao Sistema de Cadastro Imobiliário e modelagem das informações referentes ao Sistema de Registro de Imóveis. As relações entre todos esses aspectos necessitam ser mantidas e vinculadas com o tempo em que ocorrem.

Partindo desse princípio, a modelagem do Cadastro e Registro é alcançada através da definição de duas entidades separadas, mas dependentes e correlacionadas, com suas informações específicas representadas por 
propriedade e proprietário, respectivamente.

A entidade "propriedade" contém informações relativas às características da parcela, incluindo as informações geográficas/geométricas. No entanto, para manter a compatibilidade com a base de dados utilizada a ser aproveitada, optou-se por manter estas características geométricas em uma entidade separada, denominada "lote", que se refere a cada parcela.

Na Figura 1 pode ser observado o Modelo de Entidade e Relacionamento que define a modelagem do banco de dados. Vale ressaltar a utilização do campo "ssqqllff" como identificador das entidades e relacionamentos referentes ao lote e propriedade.

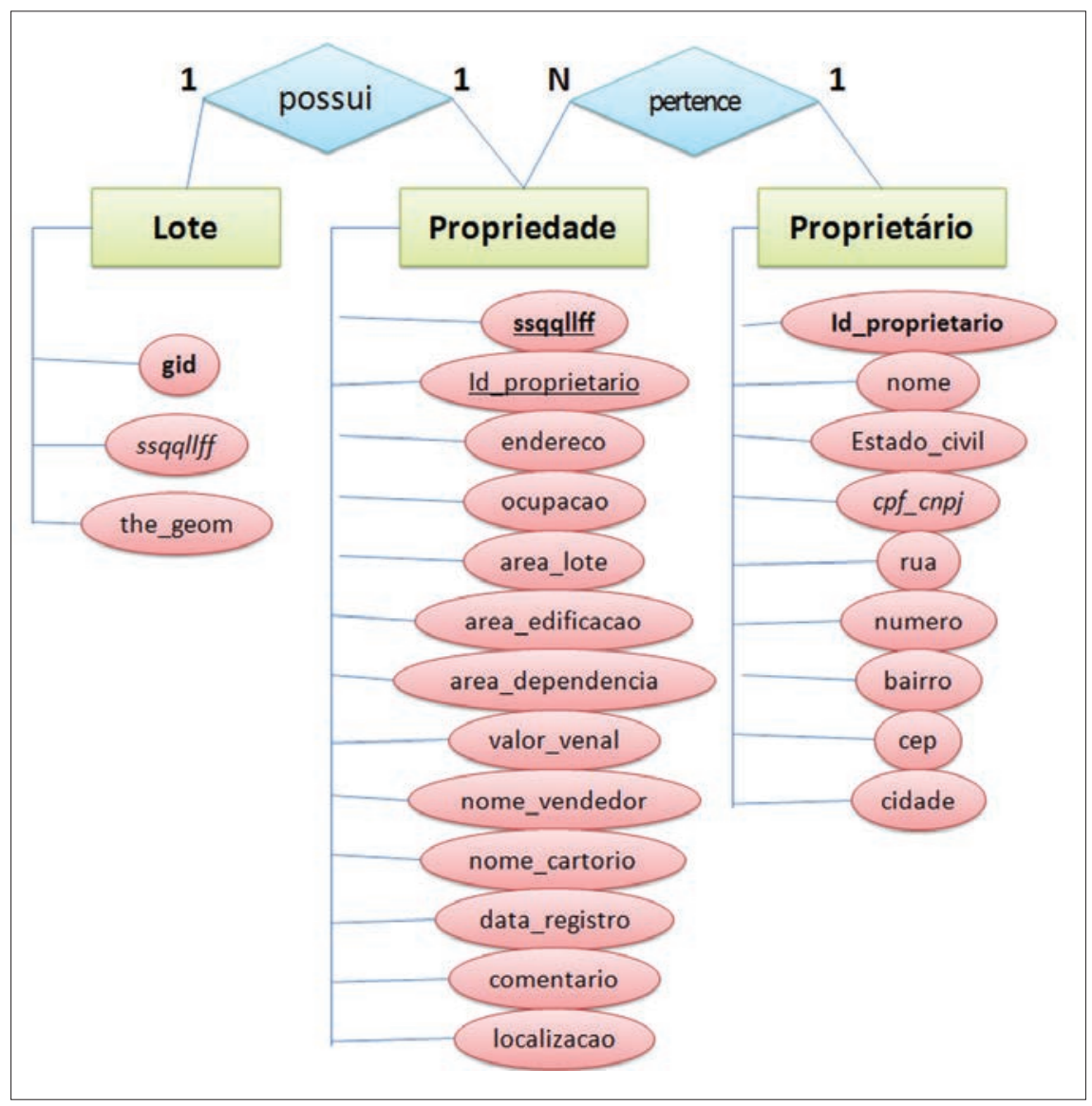

Figura 1 - Relacionamento entre as entidades 


\subsubsection{Elaboração do banco de dados}

Além da entidade "Lote" que contém as geometrias e identidades de todos os imóveis do município definiram-se outras tabelas com seus respectivos atributos, os quais podem incorporar informações referentes ao Cadastro Imobiliário e Registro de Imóveis.

Foram adicionadas ao banco de dados de Ribeirão dos Índios duas novas tabelas denominadas "Proprietário" e "Propriedade". A primeira se refere aos dados relacionados à descrição do proprietário do imóvel como nome do proprietário, endereço, CPF/CNPJ, estado civil, entre outras informações. A segunda diz respeito às características da parcela que a identifica, tornando-a única e inconfundível. Esta tabela contém dados como o identificador da parcela, endereço, data de registro, nome do antigo proprietário, dimensão da testada, profundidade, área da parcela, área da edificação, valor venal e a descrição geométrica da parcela, que inclui as coordenadas de seus respectivos limites.

É importante ressaltar que as informações que não foram obtidas pelo levantamento cadastral e que são de interesse dos sistemas de Cadastro e Registro, foram simuladas no banco de dados para enriquecer o resultado da análise e permitir testes mais condizentes com o contexto real da aplicação.

\subsubsection{Definição da Arquitetura}

Após a modelagem inicial do banco de dados, é necessário um estudo sobre os recursos a serem implementados no sistema, para que seja possível definir seus componentes e responsabilidades específicas.

Um dos recursos essenciais para este tipo de sistema é a capacidade de criação de filtros dinâmicos, configurados de acordo com a necessidade do usuário. Esses filtros são diretamente relacionados com a modelagem do banco de dados, pois, caso não seja possível a utilização e consumo de determinado dado armazenado, este dado armazenado passa a ser inútil.

Como os dados que compõem a modelagem do banco de dados podem variar de acordo com o contexto de aplicação, principalmente os dados dos proprietários e parcelas, que podem agregar dados censitários adi- 
cionais, é necessário que os filtros possam se adaptar de acordo com a modelagem utilizada, sem a necessidade de reimplementação ou geração de novos códigos do sistema.

Para isso, adotou-se uma representação em formato XML, para definir as entidades e seus respectivos campos que integram o banco de dados, ressaltando a importância da utilização da estrutura XML para permitir a integração do sistema em outros contextos. Todos os parâmetros de acesso e manipulação do banco de dados devem ser descritos no arquivo de configuração XML, como nome do banco, dados da conexão com o banco de dados e modelagem das entidades a serem consideradas.

Uma vez que a representação da modelagem é realizada em um formato conhecido, os filtros de seleção dos dados podem ser construídos dinamicamente a partir da leitura deste arquivo XML e carregados através de processamento assíncrono com JavaScript/AJAX para obtenção dos valores correspondentes.

A integração de diferentes tecnologias facilita a criação de componentes específicos responsáveis por exercer tarefas únicas no sistema. A chamada modularização do sistema é uma estratégia que agrega muitos benefícios ao processo de desenvolvimento, principalmente à fase de manutenção e atualização posterior. Neste sentido, foi adotada uma arquitetura modular, na qual cada componente é responsável por desempenhar sua funcionalidade específica e se comunicar com as camadas adjacentes.

Na Figura 2 é apresentado um diagrama que corresponde ao fluxo de interação dos módulos implementados, com diferentes tecnologias utilizadas para o desenvolvimento do sistema.

\section{RESULTADOS E DISCUSSÕES}

Como resultados deste trabalho têm-se um protótipo de SIG Cadastral, desenvolvido como um sistema Web, no qual o usuário pode analisar um determinado conjunto de dados e realizar diversas consultas de interesse tanto para os sistemas de Registro de Imóveis quanto para o Cadastro Imobiliário Urbano. 


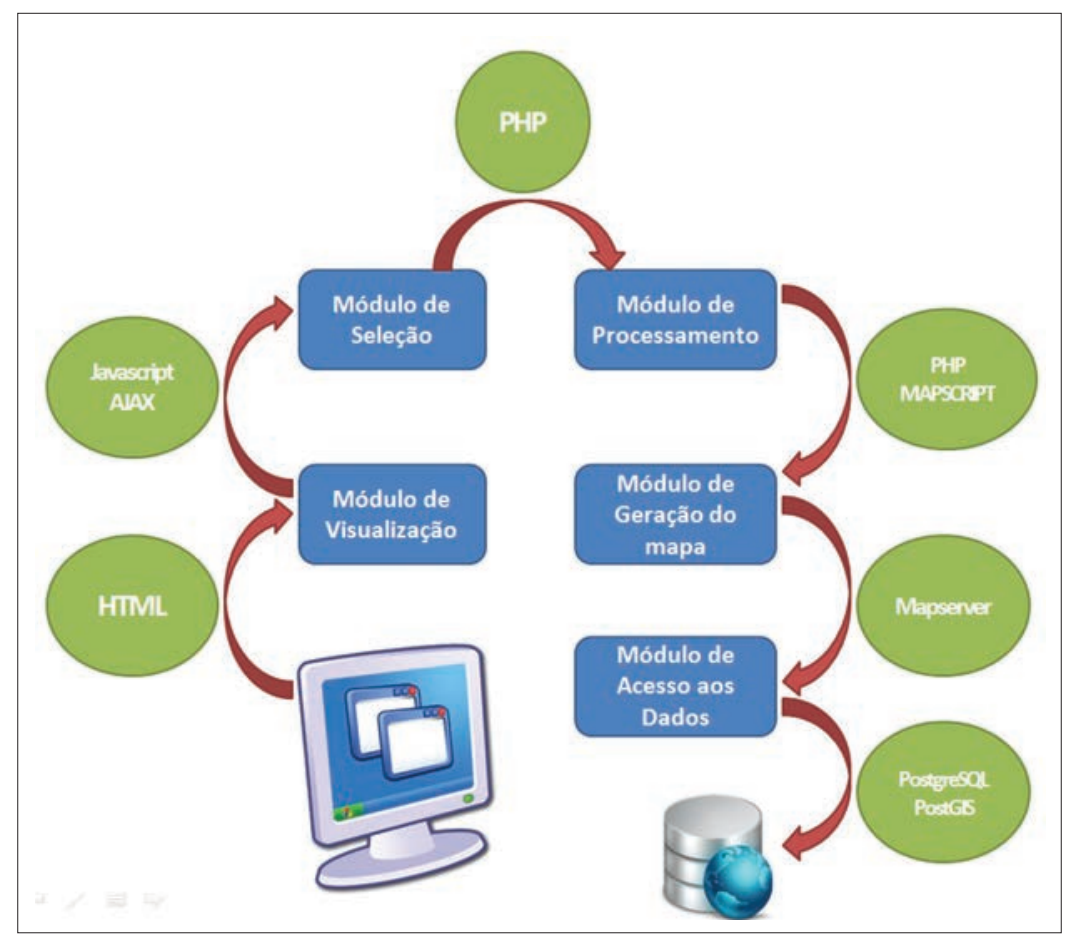

Figura 2 - Arquitetura definida para o sistema desenvolvido

O usuário tem a possibilidade de visualizar a localização de cada imóvel matriculado juntamente com suas informações cadastrais (Figura 3), verificar todos os imóveis confrontantes e ter conhecimento de quais e quantos são os imóveis registrados dentro de um setor fiscal, bem como os imóveis pertencentes a um determinado proprietário (Figura 4). Além das consultas apresentadas, pode-se buscar informações relativas a valores venais, atualizações de logradouros, bem como a verificação dos atos de desmembramentos, remembramentos e mudanças de proprietários.

Para que o cadastro seja autêntico e refita a realidade de um município é importante que não haja exclusão de informações, com exceções das situações de retificações, e sim atualizações no banco de dados baseadas na inserção de novos registros. Sendo assim, num caso em que uma parcela seja desmembrada em outras duas, as informações do antigo imóvel serão armazenadas no banco de dados e duas novas parcelas serão criadas com referencia à primeira, ou seja, a primeira deixa de existir, mas continua arquivada. 


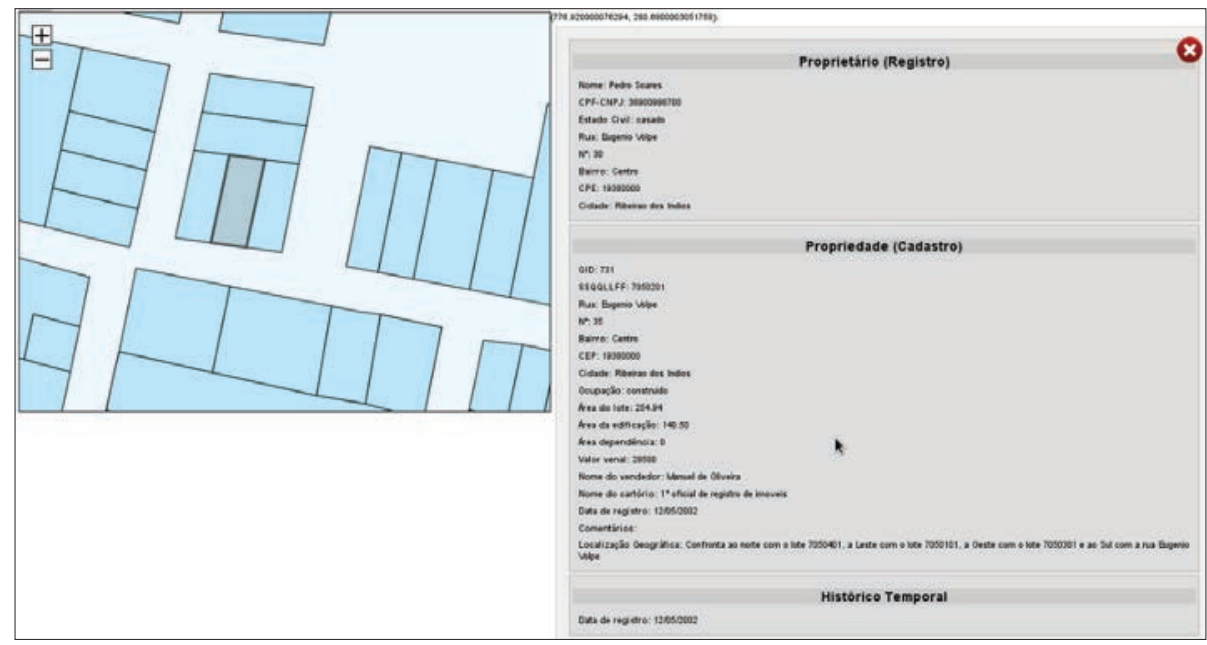

Figura 3 - Visualização das características de uma determinada parcela

Um dado importante que permite discriminar uma parcela da outra evitando a superposição de imóveis, é a sua localização geográfica por meio das coordenadas georreferenciadas que definem os limites de cada parcela. O sistema desenvolvido neste trabalho possibilita também que o usuário localize uma propriedade pelas suas respectivas coordenadas.

Existe um importante recurso que pode ser implementado em projetos futuros que é a relação do espaço temporal das informações referentes aos proprietários e parcelas e a visualização dessas no sistema. É necessário que, a partir de um novo registro, apenas as novas informações sejam visualizadas, porém as antigas devem continuar armazenadas no banco de dados, fazendo o mesmo papel dos antigos arquivos.

\section{CONCLUSÕES}

Apesar do presente trabalho ainda estar em fase de desenvolvimento, com resultados preliminares, pode se concluir que os Sistemas de Informações Geográficas são instrumentos fundamentais que podem auxiliar na integração de dados pertencentes aos sistemas cadastrais e registrais, levando em consideração que os SIG permitem o armazenamento, manipulação, análise de informações geográficas e possibilitam a visualização destas através da criação de mapas temáticos. 


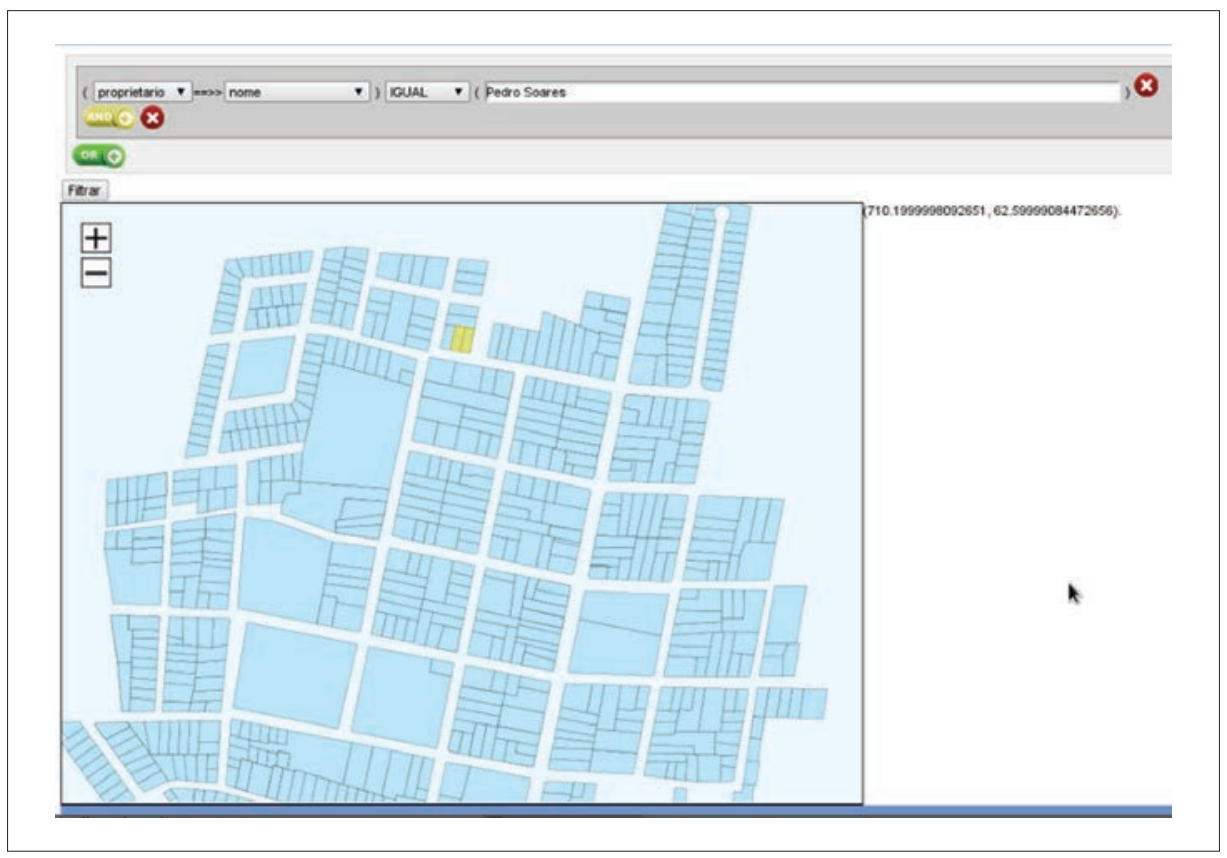

Figura 4 - Busca por parcelas pertencentes a um determinado proprietário

Além dos órgãos de Cadastro e Registro de Imóveis é importante também que a população tenha acesso a algumas informações a respeito de suas respectivas parcelas, uma vez que, o SIG Cadastral desenvolvido como um sistema Web, além de disponibilizar informações com mais agilidade e menor custo, é um sistema de fácil acesso para o usuário.

São diversas as consultas que um sistema de intercâmbio de informações entre os Sistemas de Cadastro e Registro permite. Para isso, é necessário que as informações estejam sempre atualizadas e que os dados sejam confiáveis. Respeitando essas premissas e fazendo uso das tecnologias e estratégias de implementações corretas, a disponibilização, análise e exploração das informações pode ser realizada de maneira célere e transparente, mas, sobretudo, permitido maior segurança jurídica ao processo.

Quanto a manutenção do sistema e atualização dos dados, pouco ainda se tem a concluir, uma vez que será necessário o acompanhamento do funcionamento do sistema proposto, que dependerá do próprio usuário. 


\section{AGRADECIMENTOS}

À Coordenação de Aperfeiçoamento de Pessoal de Nível Superior CAPES, pela concessão de bolsa de mestrado à um dos autores e pelo apoio financeiro que permitiu a apresentação deste artigo no evento.

\section{BIBLIOGRAFIA}

AMORIM, Amilton et al. (2008) - Cadastro técnico multifinalitário via internet: um importante instrumento de apoio ao planejamento municipal. Revista Brasileira de Cartografia, v 60/2, p. 119-125.

BRASIL. Portaria $\mathrm{N}^{\circ}$ 511, de 8 de dezembro de 2009. Portaria do Ministério das Cidades: Diretrizes para a criação, instituição e atualização do Cadastro Territorial Multifinalitário (CTM) nos municípios brasileiros, p. 75.

CARNEIRO, Andréa. Flávia (2003) - Cadastro Imobiliário e Registro de Imóveis. Instituto de registro imobiliário do Brasil. Porto Alegre.

DALE, Peter. F. \& MCLAUGHLIN, John. D. (1990) - Land information management: an introduction with special reference to cadastral problems in third world countries. Reprinted (with correction). Oxford. Oxford University Press, p. $10-15$.

DATE, Christopher. J. (2000) - Introdução aos Sistemas de Bancos de Dados. Rio de Janeiro, Editora Campus.

FIG - Federação Internacional dos Geômetras (2010). Comissão 7. Statement on the cadastre. Disponível online no endereço url: https://www.fig.net/ commission $7 /$ reports/cadastre/statement_on_cadastre.html. (acesso em 9 de março, 2013).

FILHO, Jugurta. Lisboa (1995) - Introdução ao SIG - Sistemas de Informações Geográficas. Curso de Pós Graduação em Ciência da Computação, Universidade Federal do rio Grande do Sul - UFRS. Porto Alegre, RS. Disponível online no endereço url: http://www.dpi.ufv.br/ jugurta/papers/ ti.pdf. (Acesso em 10 de Fevereiro, 2014)

GONÇALVES, Rômulo. Parma (2008) - Modelagem conceitual de banco de dados geográfico para Cadastro Técnico Multifinalitário em municípios de pequeno e médio porte. Viçosa - MG, Brasil. Disponível online no endereço url: http:// www.tede.ufv.br/tedesimplificado/tde_busca/arquivo.php?codArquivo $=1446$. (acesso em 9 de Fevereiro, 2014).

JACOSKI, Claudio. Alcides et al. (2006) - Projeto de Cadastro Técnico Multifinalitário para aplicação no Projeto SIG Chapecó. Congresso Brasileiro de Cadastro Técnico Multifinalitário. Florianópolis - SC, Brasil. 
KINBERGER, Michaela \& PUCHER, Alexander (2005) - Open Source GIS als Alternative im Desktop-Bereich - Evaluation freier Software im Bereich Geoinformation. In: CORP 2005. Wien, Österreich.

LARSSON, Gerhard. Land registration and cadastral systems. Reprinted. England, UK, Longman Group, 1996 p. 19-22.

LUACES, Miguel Rodriguez et al. (2004) - A generic framework for GIS applications. In Proceedings of the 4th international conference on Web and Wireless Geographical Information Systems (W2GIS'04). Springer-Verlag, Berlin, Heidelberg, 94-109. Disponível online no endereço http://dx.doi. org/10.1007/11427865_8 (acesso em 26 de Fevereiro, 2014)

MALAMAN, Carolina. Scherrer \& AMORIM, Amilton (2010) - Utilização do software gvSig no Cadastro Técnico Multifinalitário do município de Ribeirão dos Índios - SP. In: Congresso Brasileiro de Cadastro Técnico Multifinalitário - COBRAC. p. 1-10.

MIRANDA, José. Iguelmar (2002) - Diretivas para disponibilizar mapas na Internet. Campinas: Embrapa. Série documentos.

MIRANDA, José. Iguelmar \& SOUZA, Kleber. Xavier. Sampaio (2003) - Como publicar mapas na Web. In: Congresso Brasileiro de Sensoriamento Remoto, 11, 2003, Belo Horizonte: INPE. p. 349-355.

NASCIMENTO, Jairon. Alcir. Santos. do \& MAURO, Cláudio. Antônio. de (2006) - A sustentabilidade ambiental urbana a partir de uma perspectiva espacial: o caso das cidades da Amazônia brasileira. Mercator - Revista de Geografia da UFC. Numero 09.

OGC, Open Geospatial Consortium. Disponível online no endereço url: http:// www.opengeospatial.org/. (acesso em 15 de Fevereiro, 2014).

PARMA, Gabriel. Cremona (2006) - Mapas Cadastrais na Internet: Servidores de mapas. In: Congresso Brasileiro de Cadastro Técnico Multifinalitário. Florianópolis. Anais.

PHILIPS, Jürgen (1996) - Os dez mandamentos para um cadastro moderno de bens imobiliários. In: Congresso de Cadastro Técnico Multifinalitário, 2. Florianópolis. Anais. p. $170-183$.

PIEROZZI, Ivo. Jr et al. (2006) - Geotecnologias e geoinformação para a gestão territorial municipal. Infogeo. Curitiba, PR. n.41. p.23-24.

POSTGRESQLBR: PostgreSQL. Brasil. Disponível online no endereço url: http:// www.postgresql.org.br/. (acesso em 09 Fevereiro, 2014).

SCHMIDT, Manuela \& WEISER, Paul (2012) - Web Mapping Services: Development and Trends. In: Michael Peterson (Ed.). Online Maps with APIs and WebServices. Springer Berlin Heidelberg, 2012, 13-21. Disponível online no endereço http://link.springer.com/chapter/10.1007\%2F978-3-642-27485-5_2 (acesso em 26 de Fevereiro, 2014) 
QUEIROZ, Juliano (2007) - SGBD: O que é? Disponível online no endereço url: http://espacoinfo.net/o-que-e-sgbd-bd-ii/. (acesso em 09 de Abril, 2013).

RAMBO, Jacir. Antonio (2011) - Contribuição jurídica para a retificação administrativa georreferenciada de Imóvel Urbano nos Registros de Imóveis brasileiros. Dissertação (Mestrado em Engenharia Civil) - Universidade Federal de Santa Catarina. UFSC. Florianópolis - SC.

SASS, Glaucia. Gabriel \& Amorim Amilton (2013) - Análise temporal a partir do Cadastro Territorial Multifinalitário. Revista Brasileira de Cartografia. No 65/2: 383-291. Sociedade Brasileira de Cartografia, Geodésia, Fotogrametria e Sensoriamento Remoto. ISSN: 1808-0936

WILLIANSON, Ian et. al. (2010) - Land Administration for Sustainable Development. 1st ed. Redlands - California, Esri Press. p. 1-20. 
Série Documentos

Imprensa da Universidade de Coimbra

Coimbra University Press

2015

- U M

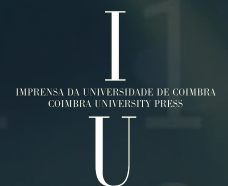

